\title{
Ameloblastic Fibro-Odontoma in a 4-Year-Old Boy
}

\author{
Mehdi Ghandehari-Motlagh, ${ }^{1}$ Zahra Khosravi, ${ }^{1, *}$ Ghasem Meighani, ${ }^{1}$ and Yahya Baradaran- \\ Nakhjavani ${ }^{1}$ \\ ${ }^{1}$ Department of Pedodontics, Dentistry School, Tehran University of Medical Sciences, Tehran, IR Iran \\ ${ }^{*}$ Corresponding author: Zahra Khosravi, Department of Pedodontics, Dentistry School, Tehran University of Medical Sciences, Tehran, IR Iran. Tel: +98-9359344933, Fax: +98-2188003880, \\ E-mail: zahrakhosrav@gmail.com
}

Received 2015 June 29; Revised 2015 October 27; Accepted 2015 November 23.

\begin{abstract}
Introduction: Ameloblastic fibro-odontoma (AFO) is defined as a benign odontogenic tumor with slow growing behavior. Its prevalence is rare. AFO is characterized by histologic features of ameloblastic fibroma (AF) with the formation of enamel and dentine.

Case Presentation: This is a case report of AFO accompanied with a number of impacted deciduous teeth and its management in a 4-year old boy. Examination of oral cavity revealed an extensive swelling from midline to left deciduous maxillary first molar, covered with normal mucosa. Radiographic examination showed a well-defined mixed radiolucent-radiopaque lesion that extended horizontally from midline to mesial border of the left maxillary primary first molar and vertically from alveolar crest to the floor of nose. The differential diagnosis was odontoma (ameloblastic fibro-odontoma, complex odontoma). Surgical enucleation and curettage was performed under general anesthesia. Histopathologic sections show bone trabeculae in marrow spaces. There was myxoid matrix in some spaces which contained odontogenic epithelial cells. These findings led to diagnosis of AFO. No sign of recurrence has been observed during the 12-month followup period.

Conclusion: Although AFO is a rare tumor, it is more prevalent in children's jaw. Conservative surgical treatment allowed the normal development of teeth.
\end{abstract}

Keywords: Ameloblastic Fibro-Odontoma, Odontogenic Tumor, Odontoma

\section{Introduction}

Ameloblastic fibro-odontoma (AFO) is defined as a mixed odontogenic tumor in which both odontogenic epithelium and ectomesenchyme are proliferated and dental hard tissue formation can be seen as its consequence. Prevalence of AFO is relatively rare, about 3.1\% of all odontogenic tumors $(1,2)$. Affected patients are generally between 8 to 12 years, and no tendency to gender or anatomic position was reported, although some articles report a slight tendency for posterior mandible involvement $(2,3)$. Most cases defined as painless swelling may be detected to be the result of tooth eruption failure (4, 5). Radiographic view of AFO consists of a well-defined unilocular or multilocular radiolucent lesion that contains part of irregular radiopaque particles (6). Because of these large mineralized parts, it is impossible to differentiate the AFO from complex odontoma through radiographic examination (7). Histopathologic feature of AFO is characterized by cords, strands and islands of odontogenic epithelium submerged in embryonic connective tissue that simulate rudimentary dental pulp $(2,4)$. For treatment of AFO conservative enucleation followed by curettage is recommended (8). Generally, the prognosis of AFO is reported as excellent (9). The purpose of this article is to describe a case of AFO that affected the left maxillary region of a patient that was treated with surgical approach without any sign of alteration or recurrence after 12 months of follow-up.

\section{Case Presentation}

The parents of a 4-year-old boy referred to the pediatric dentistry, school of dentistry, Tehran university of medical sciences, on June 2014 complaining of missing teeth. According to patient's mother, since she noticed the swelling, her son was visited immediately by a dentist who referred him to the dental school. The patient's past medical history was unremarkable. He had healthy parents and there was no history of systemic diseases in his family. A general physical examination showed no abnormality and his mother didn't mention any history of trauma. Extra-oral examination showed a painless swelling on the left anterior maxilla, with firm consistency on the palpation, which caused little facial asymmetry. The swelling was asymptomatic and covered with healthy normal colored skin. Intra-oral examination revealed a vestibular, non-tender and firm consistency swelling and expansion of buccal cortical plate that was covered with normal mucosa. The panoramic radiograph

Copyright (C) 2016, Growth \& Development Research Center. This is an open-access article distributed under the terms of the Creative Commons Attribution-NonCommercial 4.0 International License (http://creativecommons.org/licenses/by-nc/4.0/) which permits copy and redistribute the material just in noncommercial usages, provided the original work is properly cited. 
and CT scan (Axial section) revealed an expansile, mixed radiolucent-radiopaque lesion with well-defined corticated border. There was a radiolucent rim and scattered foci of calcified particles which contained several radiopaque bodies of varying sizes and shapes with density similar to enamel or dentin in the left anterior maxilla. The lesion extended horizontally from midline to mesial border of left maxillary primary first molar and vertically from alveolar crest to the floor of the nose (Figure $1 \mathrm{~A}$ ). The provisional diagnosis was complex odontoma or AFO. Excisional biopsy and careful curettage of surgical cavity and removal of impacted left primary central and lateral incisors was performed. The specimens fixed in formalin and submitted for histopathological assessment consisted of 2 pieces of bony tissue, each one having rhomboid-shape measuring $3 \times 2 \times$ $1 \mathrm{~cm}$ and one paper-tiny piece measuring $2 \times 3 \mathrm{~cm}$ (Figure $1 \mathrm{~B})$. Microscopically, sections showed bone trabeculae in marrow spaces. There was myxoid matrix in some spaces which contained odontogenic epithelial cells (Figure $1 \mathrm{C}$ ). The definitive diagnosis was AFO. After 4 months, the patient received a Nance esthetic appliance (Figure $2 \mathrm{~A}$ ). During the 12 month follow up period no sign of recurrence was detected and soft tissue healing was uneventful. Complete bone healing and normal tooth eruption pattern of permanent central and lateral incisors can be seen on last follow-up radiographs (Figure $2 \mathrm{~B}$ ).
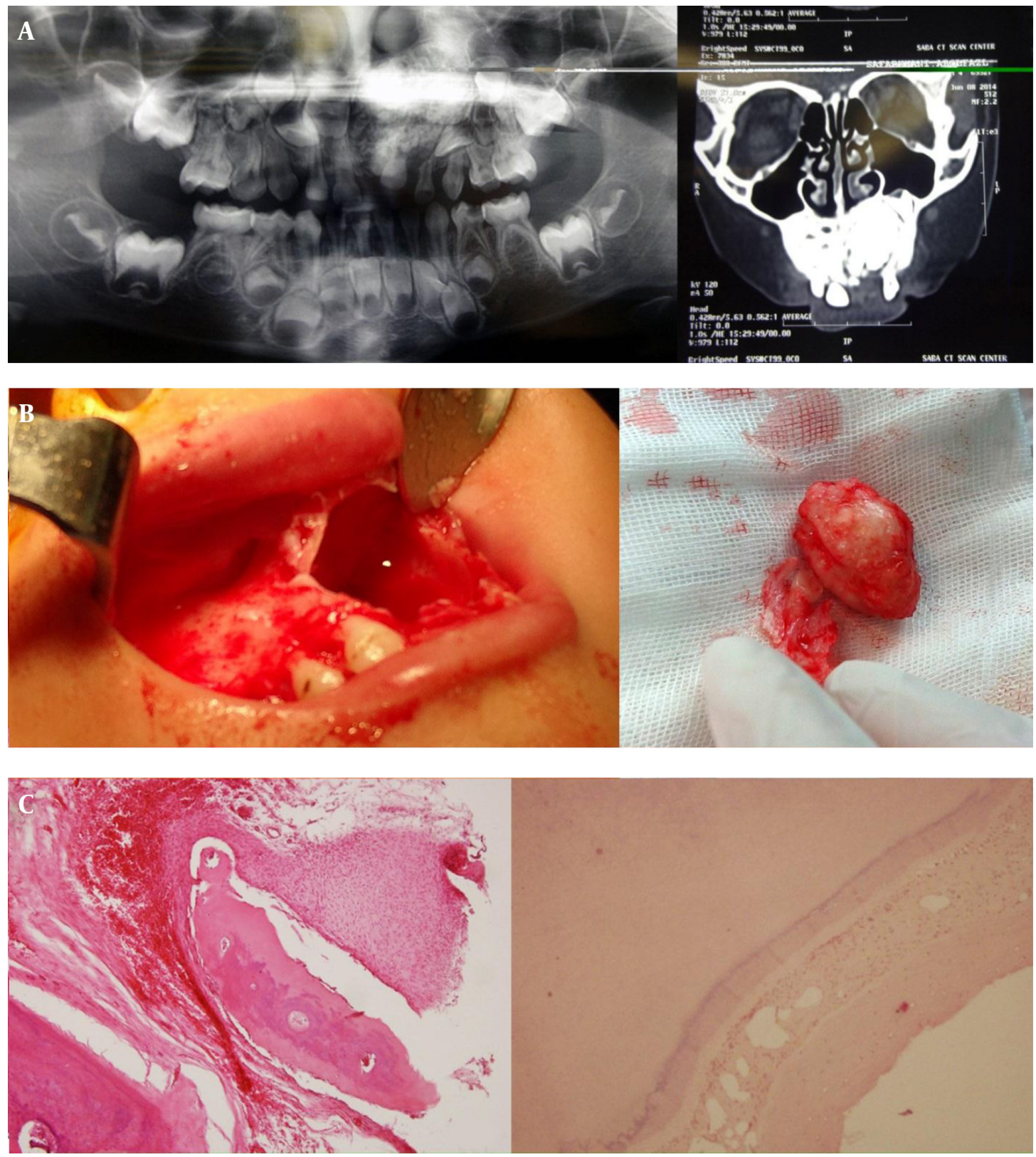

Figure 1. A, Radiographic images showing a mixed lesion in the anterior maxilla; B, surgical enucleation of the well-encapsulated lesion and its macroscopic view; C, microscopic sections showing myxoid matrix which contained odontogenic epithelial cell. 

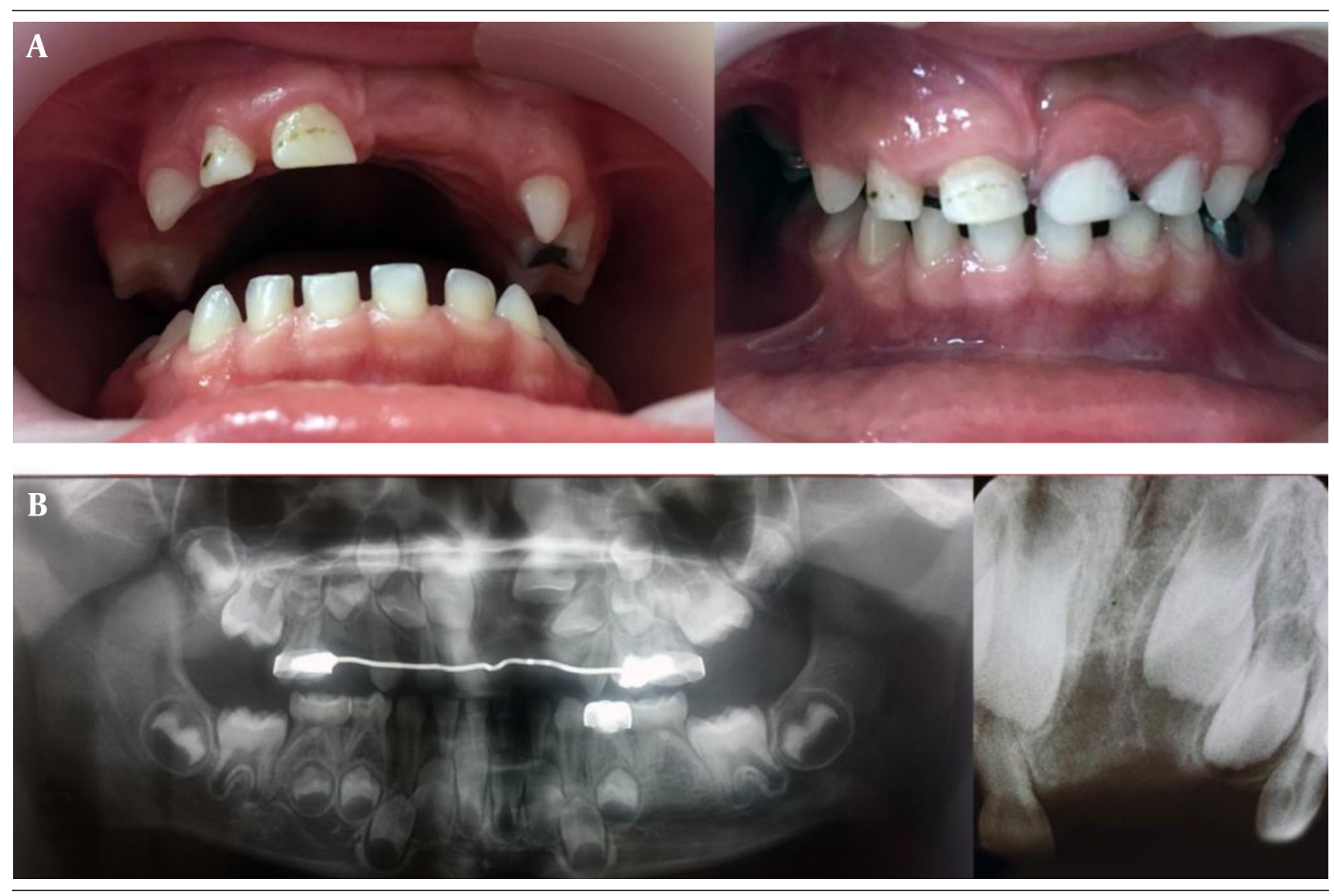

Figure 2. A, Nance esthetic appliance; B, one-year post-operative radiograph showing no sign of recurrence and position of permanent tooth germ.

\section{Discussion}

AFO is a benign tumor composed of odontogenic epithelium proliferation embedded in a ectomesenchymal tissue that resembles dental papilla, With dentin and enamel formation as a result of inductive properties of this tissue (8-10). Usually, this lesion is discovered trough routine radiographs for determining the reason of failure in tooth eruption (2). AFO is generally asymptomatic and expansion of involved hard tissue can be seen in clinical examination (4). This lesion is commonly found in the molar area, with significant tendency to mandible, affects mainly individuals under 20 years old $(1,4)$. In a review of literature, Philipsen et al. (11) found only 1 case of AFO being $>20$ years old among 86 cases that they reviewed. The average age of AFO cases at the time of diagnosis was 9 (ranging from 1 to 22 ) years, with a male/female ratio of $1.4 / 1$. Commonly, AFO was found in the posterior area of mandible, with a mandibular/maxillary ratio of 2.4 , while in our case report, lesion affected the anterior portion of maxilla in a 4-years-old boy. Radiographically, the AFO can be seen as a uni- or multilocular radiolucent lesion with a well-defined border commonly associated with a radiopaque margin (11). The ratio of radiopaque to radiolucent zones was different in each particular case; with domination of calcified structures. The lesion sometimes mimics a complex odontoma appearance (10). Surgical procedure showed a well-circumscribed tumoral mass that contains several amounts of small irregular calcified masses. Because of benign behavior of this tumor conservative and careful surgical approach is appropriate $(4,6,12)$. Sometimes, the differentiation between AFO and developing complex odontoma is impossible. However, the presence of great amount of dental hard tissue consisting of enamel, dentin, and cementum-like components organized in a haphazard pattern favors diagnosis of a complex odontoma. In addition, the differential diagnosis between AFO and odontoameloblastoma is critical. In this lesion, the epithelial component is typical of ameloblastoma and the fibrous stroma shows cellular myxoid tissue adjacent to the dental calcified tissues (1). Based on the amount of histologic differentiation, ameloblastic fibrodentinoma (AFD) is defined by some researchers as a stage between the ameloblastic fibroma (AF) and AFO (13). Histomorphologic appearance of AFO is similar to the AFD. The difference is that the AFO is specified by both enamel matrix producation and osteodentin or dentin-like deposits $(7,11)$. In contrast to AFO, AF has no signs of dental hard tissues formation (2) and potential for recurrence and malignancy exists $(3,14)$. For evaluating the nature and behavior of lesions, age of the patient and tumor's size are the most important factors that must be considered at initial detection phase. In our 
case, histopathologic assessment showed typical specification of both ectomesenchymal and epithelial components which led to diagnosis of AFO. AFO is treated with conservative surgical approach $(12,15)$. Recurrence of AFO is rare, and most recurrences are related to incomplete surgical removal (16). A recent case of AFO was treated with enucleation and impacted lower left first permanent molar was preserved and complete eruption was reported without any sign of recurrence (17). Another report, confirmed that a conservative enucleation and curettage in conjunction with copious irrigation was successful to prevent recurrences (16). Due to possible increased rate of recurrence, there is no consensus regards to tooth maintaining after AFO removal (12). However, some clinical reports achieve success without dental management followed by tooth eruption with no sign of recurrence (17). In summary, we report a case of an AFO affecting a 4-year-old boy involving the left anterior side of maxilla. In this case, enucleation and curettage was used as treatment of choice.

\section{References}

1. Nelson BL, Thompson LD. Ameloblastic fibro-odontoma. Head Neck Pathol. 2014;8(2):168-70. doi: 10.1007/s12105-013-0501-9. [PubMed: 24197722]

2. Nascimento JE, Araujo LD, Almeida LY, De Paula AM, Bonan PR. Ameloblastic fibro-odontoma: a conservative surgical approach. Med Oral Patol Oral Cir Bucal. 2009;14(12):e654-7. [PubMed: 19680188]

3. Gyulai-Gaal S, Takacs D, Szabo G, Suba Z. Mixed odontogenic tumors in children and adolescents. J Craniofac Surg. 2007;18(6):133842. doi:10.1097/scs.0b013e3180a7730f. [PubMed:17993878]

4. Buchner A, Kaffe I, Vered M. Clinical and radiological profile of ameloblastic fibro-odontoma: an update on an uncommon odontogenic tumor based on a critical analysis of 114 cases. Head Neck Pathol. 2013;7(1):54-63. doi: 10.1007/s12105-012-0397-9. [PubMed: 23001451]

5. De Riu G, Meloni SM, Contini M, Tullio A. Ameloblastic fibroodontoma. Case report and review of the literature. J Cranio- maxillofac Surg. 2010;38(2):141-4. doi:10.1016/j.jcms.2009.04.009. [PubMed: 20185068]

6. Gantala R, Gotoor SG, Kumar RV, Munisekhar MS. Ameloblastic fibro-odontoma. BMJ Case Rep. 2015;2015.

7. Sassi LM,Stramandinoli RT, Dissenha JL. Large Ameloblastic fibroodontoma in a 3 year-old child. Routh Brazil Dent J. 2011;8(1):114-6.

8. Banihashem Rad SA, Mortazavi H, Eshghpour M, Salehinejad J, Shahakbari R. A Large Ameloblastic Fibro-odontoma of the Maxillary Sinus. Iran J Otorhinolaryngol. 2014;26(75):111-4. [PubMed: 24745000]

9. Tsuji K, Yoshida H, Watanabe S, Yamamoto E, Yamada K, Tominaga $\mathrm{K}$, et al. A case of ameloblastic fibro-odontoma accompanied with a number of impacted teeth. Oral Maxillof Surg Med J. 2015;27(3):366-8. doi:10.1016/j.ajoms.2014.05.002.

10. Misra SR, Baskaran P, Maragathavalli G. Ameloblastic fibro-Odontoma: A case report. Int J Sport Stud. 2015;2(2)

11. Philipsen HP, Reichart PA, Praetorius F. Mixed odontogenic tumours and odontomas. Considerations on interrelationship. Review of the literature and presentation of 134 new cases of odontomas. Oral Oncol.1997;33(2):86-99. [PubMed: 9231165]

12. Surej Kumar LK, Manuel S, Khalam SA, Venugopal K, Sivakumar TT, Issac J. Ameloblastic fibro-odontoma. Int J Surg Case Rep. 2014;5(12):1142-4. doi: 10.1016/j.ijscr.2014.11.025. [PubMed: 25437658]

13. Chen Y, Li TJ, Gao Y, Yu SF. Ameloblastic fibroma and related lesions: a clinicopathologic study with reference to their nature and interrelationship. J Oral Pathol Med. 2005;34(10):588-95. doi: 10.1111/j.1600-0714.2005.00361.x. [PubMed:16202078]

14. Chen Y, Wang JM, Li TJ. Ameloblastic fibroma: a review of published studies with special reference to its nature and biological behavior. Oral Oncol. 2007;43(10):960-9. doi: 10.1016/j.oraloncology.2007.05.009. [PubMed: 17689135]

15. Atwan S, Geist JR. Ameloblastic fibro-odontoma: case report and review of the literature. J Mich Dent Assoc. 2008;90(11):46-9. [PubMed: 19102454]

16. Zouhary KJ, Said-Al-Naief N, Waite PD. Ameloblastic fibro-odontoma: expansile mixed radiolucent lesion in the posterior maxilla: a case report. Oral Surg Oral Med Oral Pathol Oral Radiol Endod. 2008;106(4):e15-21. doi: 10.1016/j.tripleo.2008.05.038. [PubMed: 18656394]

17. Reis SR, de Freitas CE, do Espirito Santo AR. Management of ameloblastic fibro-odontoma in a 6-year-old girl preserving the associated impacted permanent tooth. J Oral Sci. 2007;49(4):331-5. [PubMed: 18195518] 